\title{
Riemann-Liouville, Caputo, and Sequential Fractional Derivatives in Differential Games
}

\author{
Arkadii Chikrii \\ Ivan Matychyn \\ Glushkov Institute of Cybernetics \\ 40 Glushkov Prsp. \\ 03187 Kyiv \\ Ukraine \\ e-mail: chikrii@gmail.com \\ url: http://www.icyb.kiev.ua
}

\section{Abstract}

There exists a number of definitions of the fractional order derivative. The classical one is the definition by Riemann-Liouville [1]. The RiemannLiouville fractional derivatives have a singularity at zero. That is why differential equations involving these derivatives require initial conditions of special form lacking clear physical interpretation. These shortcomings do not occur with the regularized fractional derivative in the sense of Caputo. Both the Riemann-Liouville and Caputo derivatives possess neither semigroup nor commutative property. That is why so-called sequential derivatives were introduced by Miller and Ross [2].

In this paper we treat sequential derivatives of special form [3]. Their relation to the Riemann-Liouville and Caputo fractional derivatives and to each other is established.

Differential games for the systems with the fractional derivatives of Riemann-Liouville, Caputo, as well as with the sequential derivatives are studied. Representations of such systems' solutions involving the MittagLeffler generalized matrix functions [4] are given. The use of asymptotic representations of these functions in the framework of the Method of Resolving Functions [5, 6, 7] allows to derive sufficient conditions for solvability of corresponding game problems. These conditions are based on the modified Pontrjagin's condition [5]. The results are illustrated on a model example where a dynamic system of order $\pi$ pursues another system of order $e$.

\section{References}

[1] S.G. Samko, A.A. Kilbas, and O.I. Marichev. Fractional Integrals and Derivatives. Gordon \& Breach, Amsterdam, 1993.

[2] K.S. Miller, B. Ross. An introduction to the fractional calculus and fractional differential equations. Wiley \& Sons, New York, 1993.

[3] A.A. Chikrii, I.I. Matychyn. Representation of solutions to linear systems with fractional derivatives in the sense of Riemann-Liouville, Caputo, and Miller-Ross. Problemy Upravlen. Inform. (Translated in J. Automat. Inform. Sci.), 3, 2008 (to appear). 
[4] A.A. Chikrii, S.D. Eidelman. Generalized Mittag-Leffler matrix functions in game problems for evolutionary equations of fractional order Cybernetics and Systems Analysis, volume 36, 3:315-338, 2000.

[5] A.A. Chikrii. Conflict-Controlled Processes Kluwer Acad. Publ., Boston, London, Dordrecht, 1997.

[6] A.A. Chikrii. Game problems for systems with fractional derivatives of arbitrary order. In A. Haurie, S. Muto, L. Petrosyan, T. Raghavan, editors, Advances in Dynamic Games, volume 8, part II, pages 105-113. Birkhäuser, Boston, 2006.

[7] A.A. Chikrii. Game dynamic problems for systems with fractional derivatives. In A. Chinchuluun, P.M. Pardalos, A. Migdalas, L. Pitsoulis, editors, Pareto Optimality, Game Theory and Equilibria, volume 17, Springer, 2008. 